\title{
Cyclic Fatigue, Shaping Ability and Apical Transportation of Endodontic Rotary Instruments Made From Different Niti Alloys in Simulated S-Shaped Root Canal
}

\author{
DDS Justina Sinkevičiūtė \\ DDS Roberta Danilaitè \\ DDS, PhD Greta Lodienè \\ Lithuanian University of Health Sciences, \\ Department of Dental and Oral Pathology \\ Eivenių g. 2, LT-50161 Kaunas \\ Lithuania
}

\begin{abstract}
Summary
Aim: investigate the influence of NiTi alloy on cyclic fatigue resistance and fracture, shaping ability and apical transportation of rotary endodontic instruments in artificial S-shaped canals. Results: No instrument fractured. The greatest apical transportation was observed in the $R$ group, the least- in the GOLD group. $R$ group showed greater canal deviation at coronal and apical parts compared to other groups ( $p<0.05)$. CM group removed less resin at middle part, compared with the GOLD group. The minimal canal deviation in the apical curvature was observed in the GOLD group, while in the coronal curvature in the CM group. Conclusions: Instruments were resistant to fracture. Protaper GOLD ${ }^{T M}$ instruments caused minimal apical transportation and apical canal deviation, Hyflex® $C M$ instruments - coronal canal deviation.
\end{abstract}

Keywords: shaping ability, canal deviation, apical transportation, cyclic fatigue, s-shaped canal.

\section{Introduction}

During mechanical root canal instrumentation infected dentine should be removed and the root canal system prepared for proper disinfection and obturation (Neelakantan P, 2016). During this phase of endodontic treatment, complications may occur: instrument fracture due to cyclic fatigue or canal straightening, which could lead to the apical transportation. Mandibular teeth have a lower rate of the complications, whereas maxillary teeth are responsible for about $65 \%$ of the total mistakes in root canal treatment procedures. N. Haji-Hassani et al. (2015) identified errors during endo access cavity preparation and instrumentation phases comparing to the obturation phase. Apical transportation (perforation) represented 19\%, over instrumentation represented $2 \%$ and fractured instrument represented $1.5 \%$ of all mistakes(Haji-Hassani N, 2015). Instruments' separation can come by cyclic fatigue or/and torsional stress. These causes are not so common in straight canals, but when the anatomy is more difficult, there is a bigger probability of fracture. As much resistance possible to cyclic fatigue of the instrument is necessary for better prognosis, especially for curved canals(Hiran-us S P. S., 2016, Hiran-us S S. J., 2005, Pessoa OF, 2013, Pedullà E, 2012).

The unexpected failure of Nickel-titanium(NiTi) rotary instruments inside the root canal is a matter of serious concern, as these instruments may fracture without any visible sign of previous permanent deformation. Fracture due to cyclic fatigue occurs when the instrument does not bind but rotates freely in a curvature, generating tension/compression cycles at the point of maximum flexure until a fracture occurs(Pessoa OF, 2013). Such situation may occur when teeth with moderate to severe canal curvature are treated. Endodontic treatment of S-shaped root canals is one of the most difficult cases. This canal shape mostly appears in roots of molar teeth(Saleh AM, 2015). It is complicated to diagnose these situations by common dental radiographs, so the treatment can be started with no suspicion about this complexity(Topçuoğlu HS, 2016, Saleh AM, 2015).

NiTi rotary endodontic instruments have been used in clinical practice for more than two decades. Since then, manufacturers have significantly improved the instruments' parameters (Hiran-us S P. S., 2016, Fornari VJ, 2010). Recent thermo mechanical technologies have improved the flexibility and elasticity properties of NiTi instruments and reduced the straightening and apical transportation of root canal. Resistance to cyclic fatigue has also increased (Hiranus S S. J., 2005). Manufacturers apply new technologies in rotary endodontic files, that are based on "controlled memory" instead of classical "shape memory" wire (Shen Y, 2013). 
Although the manufacturers declare that their production is very resistant and long-lasting, there is still no standardized method for testing and improving this. Extracted human teeth are ideal material for testing, but neither hardness of dentine nor curves of canal anatomy can be standardized (Ferrara G, 2017).

The previous studies have shown that mistakes and complications during mechanical root canal instrumentation depend on characteristics of the instruments' alloy (Hiran-us S P. S., 2016, Hiran-us S S. J., 2005, Gergi R, 2010, Gutmann JL, 2012). NiTi alloy has special characteristics of shape memory and superelasticity. For example, NiTi CM wire files are extremely flexible and are made up of material with control memory, but not shape memory. R-phase wire instruments have a good super elasticity and shape memory effects. Gold wire files demonstrate flexibility and a great angle of rotation at fracture. The aim of this study was to investigate the influence of NiTi alloy on cyclic fatigue resistance, fracture, shaping ability, and apical transportation of rotary endodontic instruments in artificial S-shaped canals (Zupanc J, 2018). The null hypothesis tested in the present study is the significant difference of cyclic fatigue resistance and no significant difference of shaping ability and apical transportation between instruments made from different NiTi alloys in S-shaped canals.

\section{Materials and Methods}

Ninety simulated S-shaped root canals in clear resin blocks (Endo Training block-S, Dentsply Maillefer, Ballaigues, Switzerland) with $a 60^{\circ}$ coronal curvature (5mm radius) and $60^{\circ}$ apical curvature (4mm radius) were used in this study. All canals included the following: 0.02 taper, $16 \mathrm{~mm}$ length and $0.15 \mathrm{~mm}$ apical diameter (Pic 1).Sample size calculation before the study revealed a minimum of 15 canals in each group to support the statistical analysis(Ferrara G, 2017). The blocks were randomly assigned into three groups $(n=30)$, according to the investigated instruments: Hyflex ${ }^{\circledR} \mathrm{CM}$ (CM group), Protaper GOLD ${ }^{\mathrm{TM}}$ (GOLD group), and Twisted File ${ }^{\circledR}$ (R group).

The simulated canals were placed in a specially designed jig allowing resin block stabilization and image standardization. The static research method for cyclic fatigue was used(Pedullà E, 2012, Bhagabati N, 2012, Topçuoğlu HS, 2016, Ersoy I, 2016, Pongione G, 2012, Elnaghy AM, 2016, Hieawy A, 2015). The instrumentation time was video recorded and the pre-/post-instrumentation images were taken using a digital camera (Canon EOS 1100D, Canon Inc., Japan).
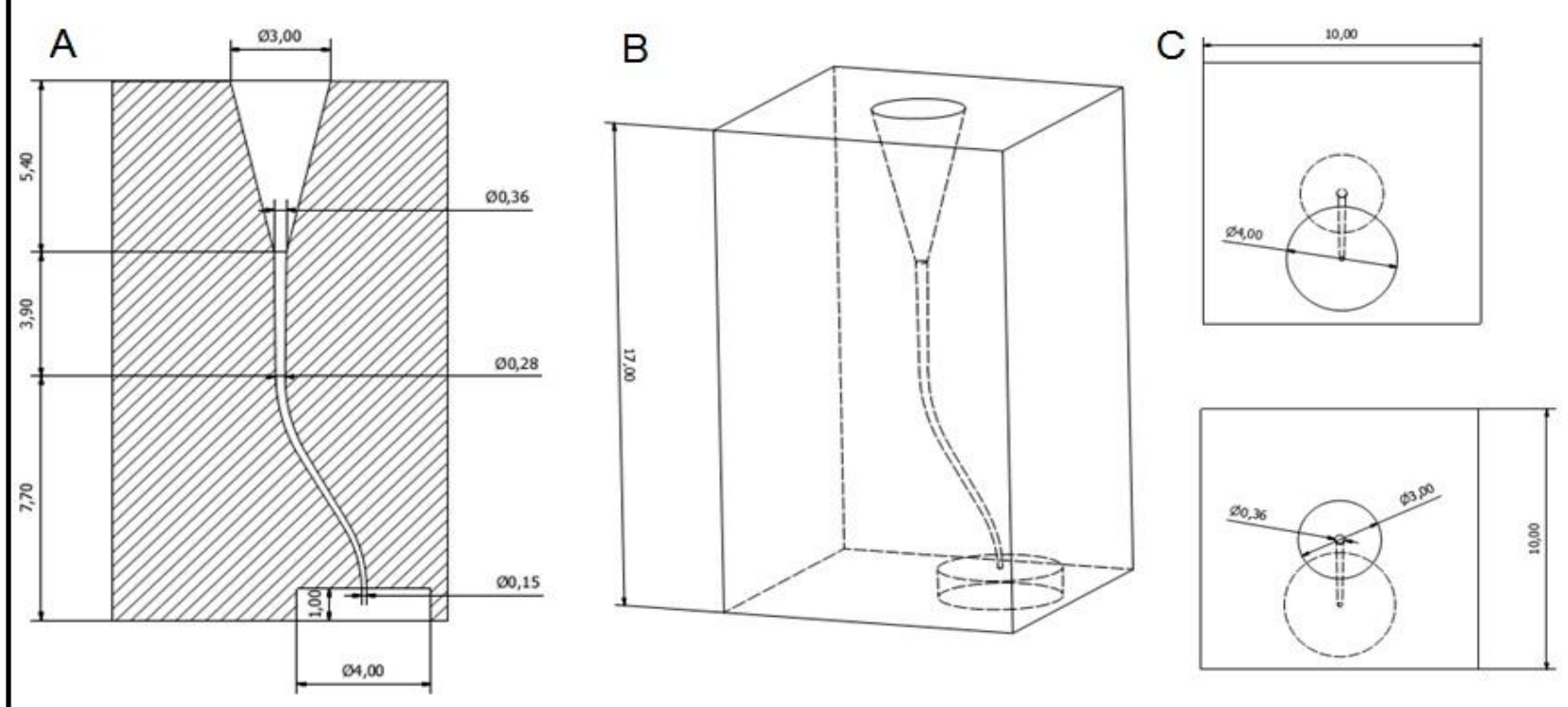

Picture 1. Resin block longitudinal section and its dimensions (A), 3D schematic view from the side (B), from the bottom and top (C) (mm).

\section{Canal preparation and instrumentation}

A glide path was established in each canal using stainless steel \#10 and \#15 (and \#20 for R group canals) K-files (Dentsply Maillefer, Switzerland) to the working length, and they were set at the apical foramen of the simulated canal. All 90 simulated canal preparations were carried out by one operator until instrument separation or after 20 minutesif separation had not occurred. A 20minute period of instrumentation was chosen because there were no previous studies that evaluated root canal shaping ability after a longer instrumentation time. 
NiTi files with asize of 40/.04 from different alloys were selected for the study because previous studies have shown, that files with a tip bigger than \#35 have a greater reduction in remaining debris(Fornari VJ, 2010). Also, an apical enlargement to size 40/.04 will allow for maximum volume of irrigation at the apical third(Brunson M, 2010). In the CM group, 25/0.08, 20/0.04, 25/0.04, 20/0.06 and30/0.04files were used. The GOLD group files were Sx, S1, S2, F1, F2, and F3. The R group files were 25/0.1, 25/0.04, 30/0.06, and35/0.06. All instrumentation was performed at a working length, except for the 25/0.08 (CM group), Sx (GOLD group), and 25/0.1 (R group) files, which were used at a $13 \mathrm{~mm}$ working length. Each canal has been instrumented using an endodontic motor Saeshin E-cube (Saeshin, Daegu, South Korea) according to the manufacturer's speed, torque and motion specifications. Each instrument was used only once, with a working time of 20 minutes. Irrigation with $3 \mathrm{ml}$ of distilled water and recapitulation with a \#10 K-file were performed after each instrument.

\section{Image analysis and canal deviation measurement}

Pre-/post-instrumentation images of simulated canals were taken under standardized conditions in order to determine the standardized position. A Canon EOS 1100D (Tokyo, Japan) camera was secured at a fixed distance $(50 \mathrm{~cm})$ from a resin blocks stage. Before instrumentation, a stainless steel \#10 K-file instrument with a silicone stopper was inserted into the canals as a scale marker (canal before instrumentation). After instrumentation, white dye (Trodat, Austria) was injected into the simulated canal to the working length in order to record the shape of enlarged canals (canal after instrumentation).

The images of pre-/post-instrumentation were superimposed into one picture using software (Adobe Photoshop CS4 Extended, Adobe System, San Jose, CA, USA) by precisely overlapping the reference points of each image (Pic. 2). The determination of the root canal deviation was modified from the S. Hiran-us et al study(Hiran-us S P. S., 2016). In total, 90 pictures were taken $(n=30$ in each group). The scale for each picture was chosen individually as a 3mmdiameter silicone stopper of the \#10 K-file instrument. The first measuring point was at the apical foramen position (L0), and the last was $7 \mathrm{~mm}$ from the apical foramen (L7), resulting in 8 measuring points for each mesial (M) and distal (D) wall of the canal. All measurements were performed perpendicularly to the canal wall. The distance between the canal walls in the pre-/post-instrumentation position was measured at $400 \%$ magnification by the same software. Data were processed by Microsoft Excel 2010 (Microsoft Office 2010, Windows 2010), and canal deviation was calculated. If the difference between the mesial and distal measurements at a given point was equal to 0 , the canal was considered non-deviated at that level. The mean value and standard deviation were calculated at each measuring point.

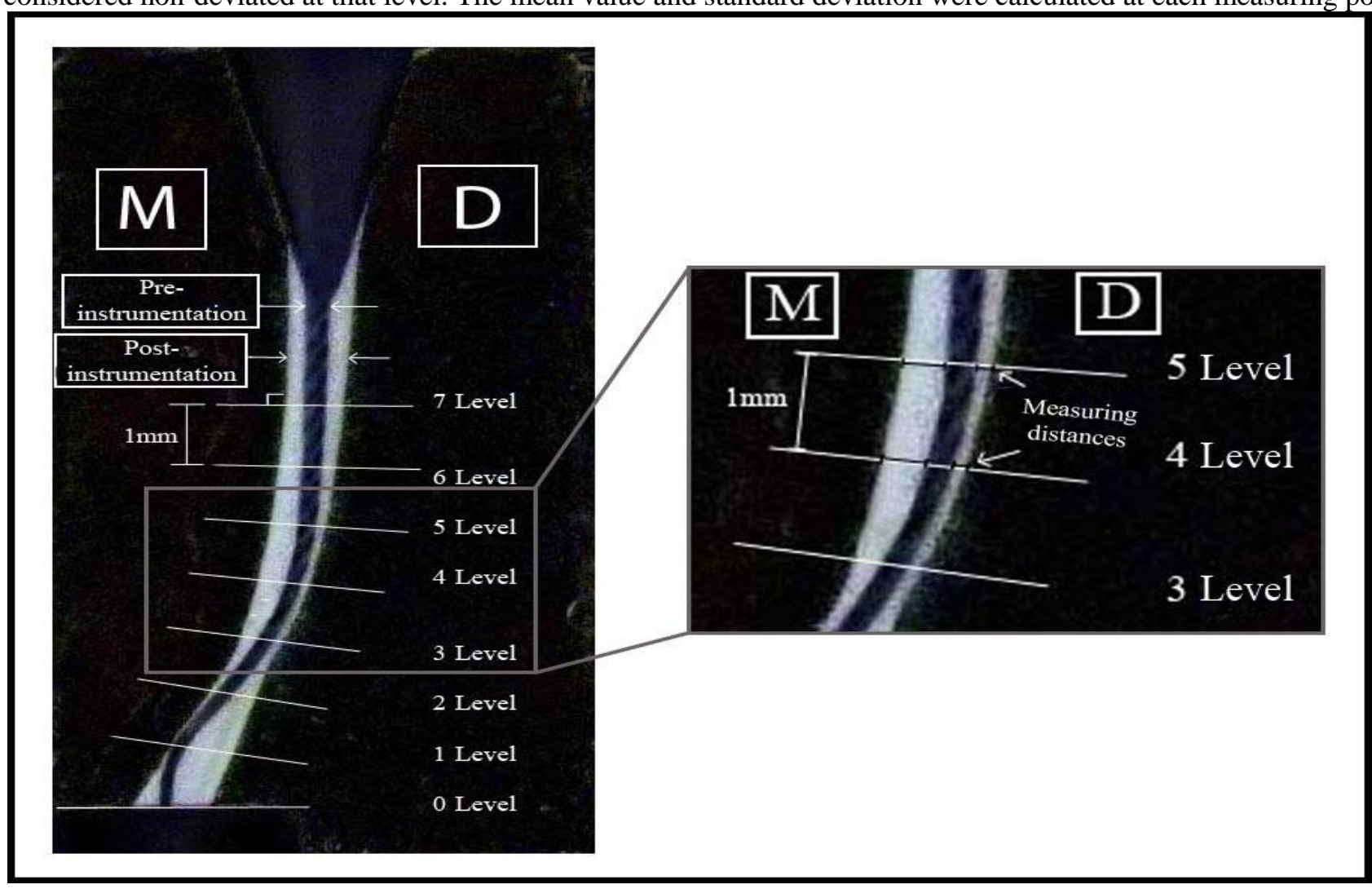

Picture 2. Pre-/post-instrumentation superimposed pictures with measuring levels (from L0 to L7). 


\section{Statistical analysis}

The data were analyzed using the Statistical Package for Social Sciences (SPSS) software (IBM SPSS Statistics 24; SPSS Inc, Chicago, IL, JAV). The Shapiro-Wilk test was performed to determine the normality of the data (if $p>0.05$ the distribution of data is significant). The differences in the distance between the mesial and distal sides at each level were compared between the three systems using the Kruskal-Wallis test and significant pairs were identified by the Mann-Whitney U-test.

\section{Results}

\section{Cyclic fatigue}

No instruments' fracture was observed during mechanical preparation in simulated S-shaped canals for 20 minutes (1200 s). The number of the instrument's rotations was significantly different because of rotational speed: 10000, 6000 and 11000 for $\mathrm{CM}$ group, GOLD group, and $\mathrm{R}$ group respectively $(\mathrm{p}<0.05)$. R group instruments performed the significantly most rotations $(\mathrm{p}<0.05)$ in 20 minutes.

\section{Canal deviation}

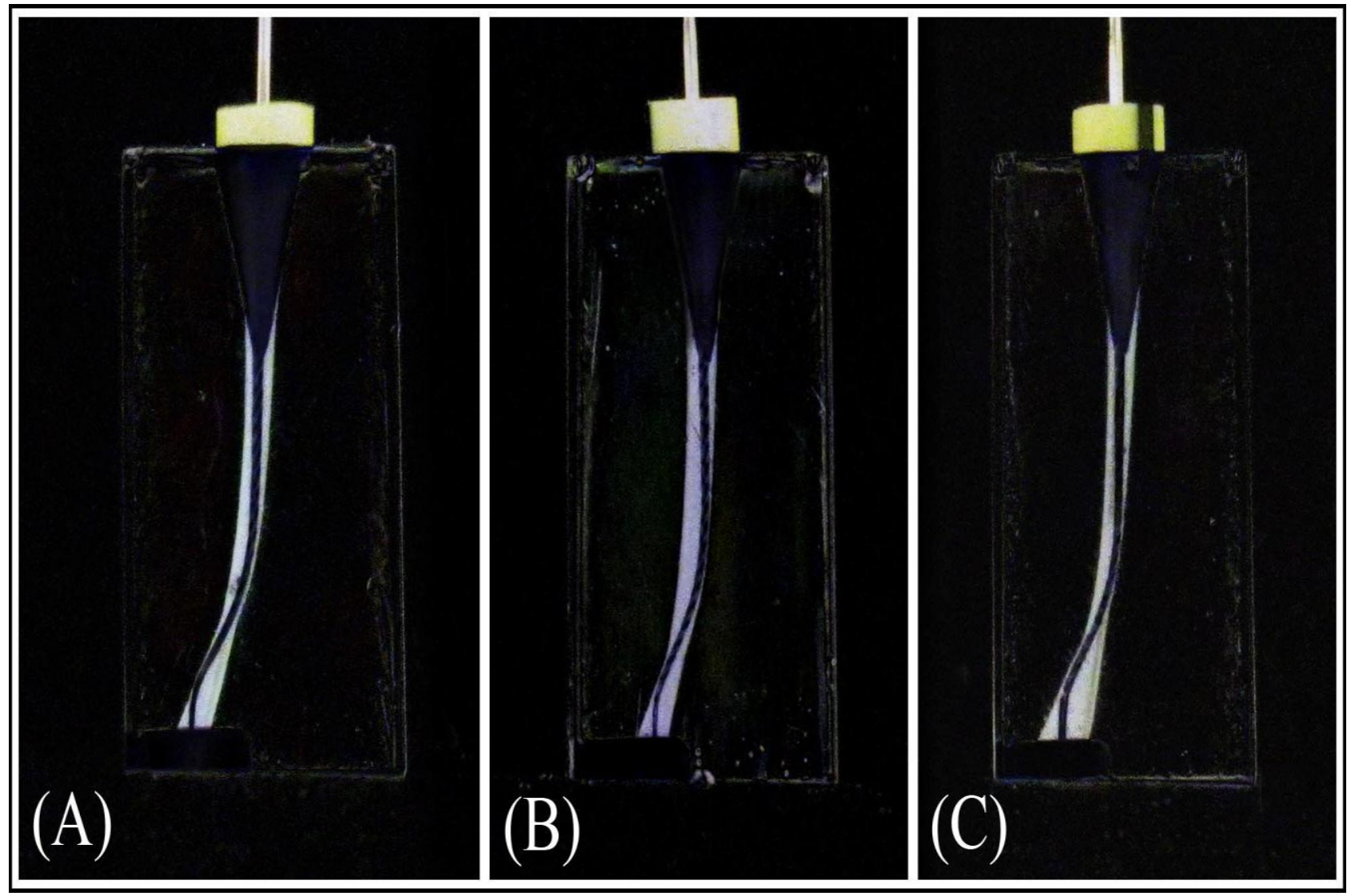

Picture 3. Pre-/post-instrumentation pictures: CM group (A), GOLD group (B) and R group (C).

After instrumentation, canal deviations were detected at almost all measuring levels in all groups. At L6 (6 mm from the apex), there was no significant difference in canal deviation between all three groups $(\mathrm{p}=0.420)$. Instruments in the $\mathrm{R}$ group showed significantly greater canal deviation at L7, L2, and L1 levels, compared to the other instruments tested $(\mathrm{p}<0.05)$ and the greatest canal deviation in the inner wall of apical curvature (distal side). The preparation of these instruments was significantly greater in the inner wall of coronal curvature (mesial side)at L5, L4, and L3 levels, compared with the GOLD group instruments $(\mathrm{p}<0.05)$ as well. CM group instruments removed significantly less resin at L5, L4, and L3 levels, compared with the GOLD group instruments ( $<<0.05)$. At levels L2 and L1, the preparation of GOLD group instruments was significantly greater than CM group instruments and showed significantly better results in apical curvature $(\mathrm{p}<0.05)$. The minimal canal deviation in apical curvature (L1-L3 levels) was observed after instrumentation with the GOLD group instruments, whereas it was observed in the coronal curvature (L4-L7 levels) with CM group instruments. 
Table 1. Canal deviation in different groups

\begin{tabular}{|l|l|l|l|}
\hline Groups & $\begin{array}{l}\text { CM group } \\
\text { Mean value } \pm \\
\text { Standard deviation }(\mathbf{m m})\end{array}$ & $\begin{array}{l}\text { GOLD group } \\
\text { Mean value } \pm \\
\text { Standard deviation } \\
(\mathbf{m m})\end{array}$ & $\begin{array}{l}\text { R group } \\
\text { Mean value } \pm \\
\text { Standard deviation } \\
(\mathbf{m m})\end{array}$ \\
\hline L7 & $0,071 \pm 0,092^{\wedge}$ & $0,071 \pm 0,141 \sim$ & $0,117 \pm 0,091^{\wedge} \sim$ \\
\hline L6 & $0,194 \pm 0,083$ & $0,218 \pm 0,123$ & $0,223 \pm 0,09$ \\
\hline L5 & $0,291 \pm 0,081^{*}$ & $0,359 \pm 0,088^{*} \sim$ & $0,299 \pm 0,066 \sim$ \\
\hline L4 & $0,279 \pm 0,056^{*}$ & $0,395 \pm 0,054^{*} \sim$ & $0,285 \pm 0,057 \sim$ \\
\hline L3 & $0,114 \pm 0,065^{*}$ & $0,246 \pm 0,06^{*} \sim$ & $0,065 \pm 0,096 \sim$ \\
\hline L2 & $-0,218 \pm 0,091^{* \wedge}$ & $-0,105 \pm 0,092^{*} \sim$ & $-0,393 \pm 0,103^{\wedge} \sim$ \\
\hline L1 & $-0,468 \pm 0,096^{* \wedge}$ & $-0,372 \pm 0,099^{*} \sim$ & $-0,782 \pm 0,113^{\wedge} \sim$ \\
\hline $\begin{array}{l}\text { L0 } \\
\text { foramen }\end{array}$ & $-0,068 \pm 0,242^{\wedge}$ & $-0,003 \pm 0,201 \sim$ & $-0,553 \pm 0,270^{\wedge} \sim$ \\
\hline
\end{tabular}

Positive values indicate the canal deviation to the mesial side(M), negative values - deviation to the distal side (D).

* - significant difference betweenCM and GOLD groups.

$\wedge$ - significant difference between CM and R groups.

$\sim$ - significant difference between GOLD and R groups.

\section{Apical foramen transportation}

The apical foramen of all sample was transported to the inner curve of the apical curvature (distal side):0.068 mm, $0.003 \mathrm{~mm}$ and $0.553 \mathrm{~mm}$ in the CM group, GOLD group and R group, respectively. The greatest apical foramen transportation was observed in the R group ( $\mathrm{p}<0.05$ ), whereas the least apical transportation was observed in the GOLD group with no significant difference compared to CM group instruments ( $\mathrm{p}>0.05)$.

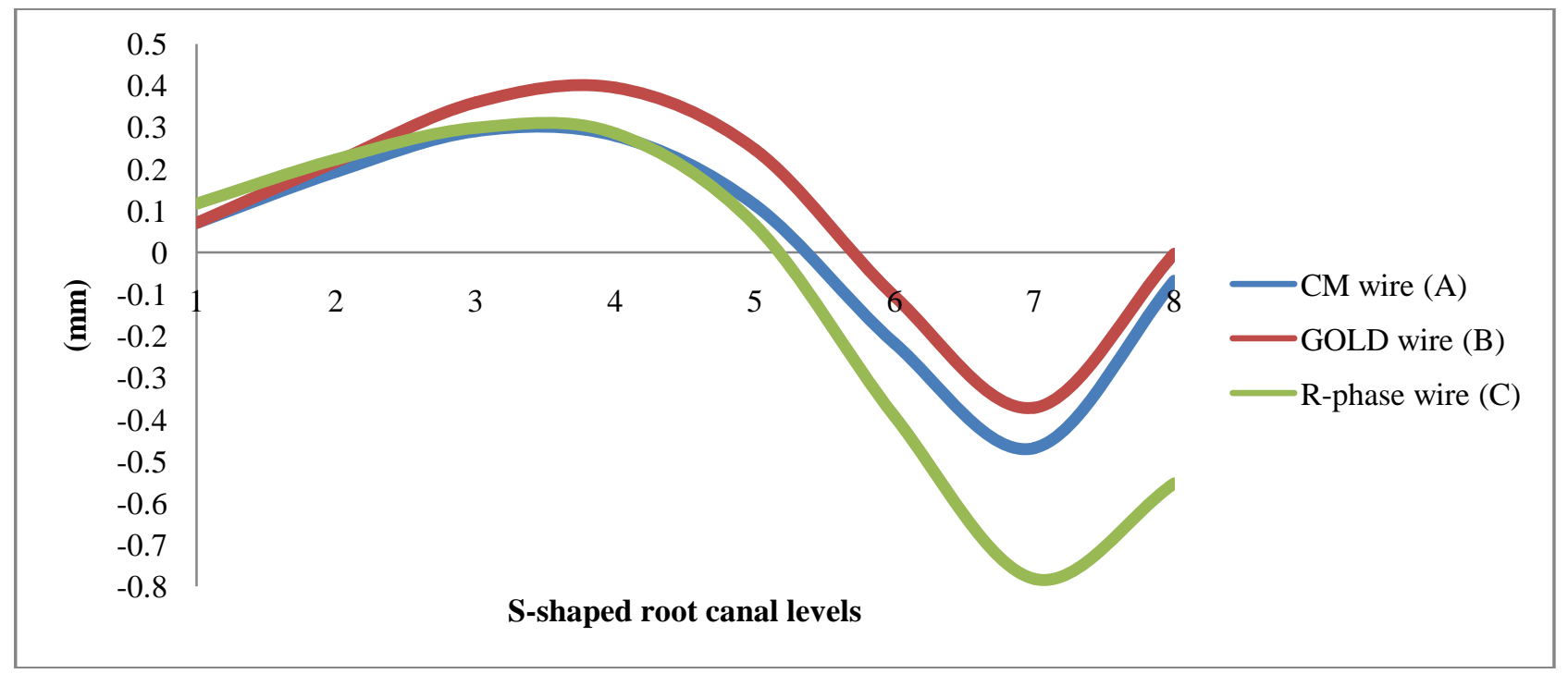

Figure 4. The direction and amount of canal transportation $(\mathrm{mm})$ at different measurement points. Positive figure values indicate the canal deviation to the mesial side (M), negative values - deviation to the distal side (D).

\section{Discussion}

Thermo mechanical treatment during the manufacturing process leads to the creation of a new type of the wire of NiTi alloy. Endodontic instruments made from NiTi controlled-memory wire, M-wire, R-phase wire, and GOLD wire represent the next generation of NiTi alloys that have better flexural fatigue resistance compared to files of similar design and size made from conventional NiTi alloy. These advantages of NiTi files for root canal cleaning and shaping decrease canal transportation and edging, reduce the risk of instrument fracture, and lead to faster and greater canal instrumentation(Shen Y, 2013).

The purpose of this study was to investigate the influence of NiTi alloy on cyclic fatigue resistance, fracture, shaping ability, and apical transportation of rotary endodontic instruments in artificial S-shaped canals. The object was instruments made from different NiTi alloys, so standardization of the study of conditions was obligatory. 
It would be better to use human teeth to make conditions more relevant to the clinical situation, but this would be more difficult to standardize due to root canal curvatures and dentin hardness. Standard resin blocks have been widely used in S-shaped root canal shaping ability studies, as it is easier to standardize the conditions and take pre- and postinstrumentation pictures, and it enables one to visualize the instrument throughout the experiment (Hiran-us S P. S., 2016, Burroughs JR, 2012, Pereira ESJ, 2012, Wu H, 2015, Reddy PJ, 2014, Saleh AM, 2015, Schafer E, 2005). The toughness of these resin blocks is less $(22 \mathrm{kgf} / \mathrm{mm} 2$ by Knoop), compared to dentine ( $68 \mathrm{kgf} / \mathrm{mm} 2$ by Knoop)(Gale WF, 2004), but more similar to stainless steel $(290-600 \mathrm{kgf} / \mathrm{mm} 2$ by Vickers), which is sometimes used in cyclic fatigue studies.

In previous studies, the root canals' instrumentation time in vitro varied from 1 to 11 minutes(Pedullà E, 2012, Bhagabati N, 2012, Topçuoğlu HS, 2016, Ersoy I, 2016, Pongione G, 2012, Elnaghy AM, 2016, Hieawy A, 2015, Hulsmann M, 2005).In this research, a long period for instrumentation (e.g., 20 minutes)was chosen, because these NiTialloys were very resistant to cyclic fatigue in previous studies(Pedullà E, 2012, Bhagabati N, 2012, Topçuoğlu HS, 2016, Ersoy I, 2016, Pongione G, 2012, Elnaghy AM, 2016, Hieawy A, 2015, Hulsmann M, 2005).However, in clinical practice, the duration of chemo mechanical preparation of root canals is shorter. Different teeth tissues have different hardness and it is not possible to predict how long the root canal will be instrumented for. It would be useful to know the results of root canal shaping ability after longer instrumentation, especially for information about instrument resistance(Hulsmann M, 2005).

Previous studies have shown that when the radius or one curvature of the root canal is smaller, the resistance to cyclic fatigue is bigger(Bhagabati N, 2012, Al-Sudani D, 2012, Ersoy I, 2016, Elnaghy AM, 2016, Hieawy A, 2015). For example, Hyflex® CM instruments performed twice as many cycles to fracture in S-shaped root canals than in single curved ones(Topçuoğlu HS, 2016, Pongione G, 2012).It is necessary to compare the results with other instrument systems. A standardized method and appliance to investigate cyclic fatigue are needed for this purpose. During the instrumentation of S-shaped canals, it was found that the centre of the canal mostly deviates in certain areas: coronal and apical curvatures. In both curvatures, the canal deviation is observed on the inner wall of the curvature(Hiran-us $\mathrm{S}$ P. S., 2016, Burroughs JR, 2012, Wu H, 2015, Saleh AM, 2015, Bonaccorso A, 2009). These findings are in agreement with the current study, where canal deviation appeared on the inner wall of the curvature in L0-L3 levels (apical curvature) and in L3-L7 levels (coronal curvature).

Size \#40 tip and 0.04 taper rotary NiTi files from different alloys were selected for the study because of the instrument's optimal size to finish the instrumentation of a root canal (Fornari VJ, 2010). It was shown, that when the NiTiHyflex ${ }^{\circledR}$ CM, Protaper GOLD ${ }^{\mathrm{TM}}$ and Twisted File ${ }^{\circledR}$ instruments are bigger, there is a weaker resistance to cyclic fatigue (Pedullà E, 2012, Bhagabati N, 2012, Ersoy I, 2016, Hieawy A, 2015). The bigger size of the apical diameter allowed more effective irrigation and removal of necrotic dentine (Fornari VJ, 2010). V. J. Fornari et al. (2010) found that instruments with size \#40 \#45 tips provided a greater reduction of remaining debris and untouched root canal walls than those with size \#30 \#35 tips at the apical third of severely curved root canals. Infected tissues in the apical part of the canal may affect the quality of treatment and the occurrence of complications (Fornari VJ, 2010). M. Brunson et al. (2010) also found that an increase in apical preparation size and taper results to maximum volume of irrigation at the apical third (Brunson M, 2010). It is impossible to clean root canals perfectly with any instrument, but an instrument with bigger apical size is a better choice to reach superior preparation of apex(Fornari VJ, 2010, Bartha $\mathrm{T}, 2006)$.

Different rotational speeds were applied for instruments in previous trials (Pedullà E, 2012, Bhagabati N, 2012, Topçuoğlu HS, 2016, Ersoy I, 2016, Pongione G, 2012). It was found that NiTi instruments made fewer cycles at higher speed (Lopes HP, 2009). For example, R-phase wire instruments made more cycles to fracture at slow rotation (Perez-Higueras JJ, 2013). Some studies have shown converse results with no relation between rotary speed and cyclic fatigue(Gao Y, 2010).In the current study, the rotational speed was different for every instrument system according to the manufacturers' recommendations, and was as follows: Twisted File ${ }^{\circledR}>$ Hyflex ${ }^{\circledR}$ CM $>$ Protaper GOLD ${ }^{\mathrm{TM}}$. There is no proven relation between rotational speed and cycles. As the highest speed was used for the Twisted File ${ }^{\circledR}$ instruments in this study, this possibly caused the greatest deviation from the canal center. It can be assumed, that the instrument speed can affect the formation of root canals (i.e., deviation from the canal center). In the E. Schafer et al. (2005) study, the authors compared the canal formation by changing the instrument's speed and torque. However, they found there was no significant difference in the formation of root canals by changing the instrumentation parameters (Schafer E, 2005). One of the mechanical preparation goals in S-shaped root canals is to preserve the natural anatomy of the canal and to minimize the apical foramen transportation. Altered canal anatomy and parameters may have a negative effect on sealing the apical third of the root canal (Wu M, 2000). However, this study showed that it is impossible to avoid apical foramen transportation in S-shaped root canals. 
The greatest apical foramen transportation was made using Twisted File ${ }^{\circledR}$ instruments, whereas the least was made using Protaper GOLD ${ }^{\mathrm{TM}}$ instruments. The manufacturer declares, that Protaper GOLD ${ }^{\mathrm{TM}}$ instruments are superior to others, not only because of their flexibility but also due to their improved non-cutting tip, which closely follows the anatomy of the root canal and thus reduces the canal deviation from center to a minimum(Specialties, 2015). It can be assumed that this led to the great results of the Protaper GOLD ${ }^{\mathrm{TM}}$ instrument (i.e., the least transportation ofthe apical foramen).

P. J. Reddy et al. (2014) compared the shaping ability of R-phase wire (Twisted File $($ ) and conventional NiTi wire (OneShape $\left.{ }^{\circledR}\right)$ instruments. R-phase wire instruments showed significantly less canal deviation from root canal center than conventional NiTi wire instruments. The cutting blades of these instruments are made by twisting the instrument and not by grinding, as seen with other tested instruments (OneShape ${ }^{\circledR}$ ).In addition, their surfaces are treated with advanced surface conditioning, which leads to more effective root canal formation(Reddy PJ, 2014).However, these results contradict the current study, where Twisted File $®$ instruments showed the biggest canal deviation from root canal center. These instruments were introduced in 2008(Honardar K, 2014), where as Hyflex ${ }^{\circledR}$ CM and Protaper GOLD $^{\mathrm{TM}}$ were introduced in 2011 and 2015, respectively (Hieawy A, 2015, Pongione G, 2012). From 2008 years to today, manufacturers have greatly improved NiTi wires for rotary endodontic instruments in all aspects.

Hyflex® $\mathrm{CM}$ and Protaper GOLD ${ }^{\mathrm{TM}}$ instruments showed significantly less canal deviation from root canal center in all canal levels and less apical foramen transportation. Comparing instrumentation at different levels, the least apical foramen transportation and apical curvature canal deviation from root canal center were achieved by the Protaper GOLD $^{\mathrm{TM}}$ instruments group. The Hyflex® $\mathrm{CM}$ instruments group showed the least canal deviation at coronal curvature. One of the factors to ensure high-quality endodontic treatment is the proper mechanical preparation of the canal and, in particular, the formation of the apical part(Fornari VJ, 2010). Since the proper formation of S-shaped root canals is considered a challenge in endodontic treatment, instruments with minimally altered apical curvature anatomy and apical foramen place are considered to be the most suitable for dental treatment with S-shaped root canals.

Within the limits of this investigation, it can be concluded that the Protaper GOLD ${ }^{\mathrm{TM}}$ and Hyflex® ${ }^{\circledR}$ Minstruments performed similarly regarding the straightening of curved root canals and apical transportation in a simulated S-shaped root canal's resin block.All tested instruments were resistant to the cyclic fatigue. In addition, Protaper GOLD ${ }^{\mathrm{TM}}$ instruments caused minimal apical transportation, suggesting that they can be a valid alternative for root canal instrumentation.

The material support of Dentsply/Maillefer (Ballaigues, Switzerland), Coltene/Whaledent (Altstetten, Switzerland) and SybronEndo (Orange, CA, USA) are gratefully acknowledged.

Funding information: Materials for this research were provided by Dentsply Maillefer, Coltene group and Kerr companys.

\section{References}

Al-Sudani D, G. N. (2012). Cyclic fatigue of nickel-titanium rotary instruments in a double (S-shaped) simulated curvature. J Endod, 38, 987-989.

Bartha T, K. M. (2006). Extended apical enlargement with hand files versus rotary NiTi files. Part II. Oral Surg Oral Med Oral Pathol Oral Radiol Endod, 102, 692-627.

Bhagabati N, Y. S. (2012). An in vitro cyclic fatigue analysis of different endodontic nickel-titanium rotary instruments. J Endod, 38, 515-518.

Bonaccorso A, C. G. (2009). Shaping Ability of Four Nickel-Titanium Rotary Instruments in Simulated S-Shaped Canals. J Endod, 35, 883-886.

Brunson M, H. C. (2010). Effect of apical preparation size and preparation taper on irrigant volume delivered by using negative pressure irrigation system. J Endod, 36, 721-724.

Burroughs JR, B. B. (2012). Shaping Ability of Three Nickel-Titanium Endodontic File Systems in Simulated S-shaped Root Canals. J Endod, 38, 1618-1621.

Elnaghy AM, E. S. (2016). Mechanical properties of ProTaper Gold nickel-titanium rotary instruments. Int Endod J, 49(11), 1073-1078.

Ersoy I, K. E. (2016). Comparison of cyclic fatigue resistance between different NiTi instruments with $4 \%$ taper. Microsc Res Tech, 79(5), 345-348.

Ferrara G, T. S. (2017). Comparative evaluation of the shaping ability of two different nickel-titanium rotatory files in curved root canals of extracted human molar teeth. J Investig Clin Dent. 
Fornari VJ, S.-S. Y.-N. (2010). Histological evaluation of the effectiveness of increased apical enlargement for cleaning the apical third of curved canals. Int Endod J, 43, 988-994.

Gale WF, T. T. (2004). Mechanical properties of metals and alloys (Vol. 22). Smithells metals reference book. 8th ed. Butterworth-Heinemann: Elsevier.

Gao Y, S. V. (2010). Effects of raw material and rotational speed on the cyclic fatigue of ProFile Vortex rotary instruments. J Endod, 36, 1205-1209.

Gergi R, R. J. (2010). Comparison of Canal Transportation and Centering Ability of Twisted Files, Pathfile-ProTaper System, and Stainless Steel Hand K-Files by Using Computed Tomography. J Endod, 36, 904-907.

Gutmann JL, G. Y. (2012). Alteration in the inherent metallic and surface properties of nickel-titanium root canal instruments to enhance performance, durability and safety: a focused review. Int Endod J, 45, 113-128.

Haji-Hassani N, B. M. (2015). Frequency of Iatrogenic Errors through Root Canal Treatment Procedure in 1335 Charts of Dental Patients. (7), 14-17.

Hieawy A, H. M. (2015). Phase transformation behavior and resistance to bending and cyclic fatigue of ProTaper Gold and ProTaper universal instruments. J Endod, 41(7), 1134-1138.

Hiran-us S, P. S. (2016). Shaping Ability of ProTaper NEXT, ProTaper UNIVERSAL and iRace files in simulated Sshaped canals. $42,32-36$.

Hiran-us S, S. J. (2005). Canal Aberrations Promoted by Three Nickel-Titanium Rotatory Instruments in Simulated SShaped canals. J Endod, 31, 373-375.

Honardar K, A. H. (2014). Cone-beam Computed Tomographic Assessment of Canal Centering Ability an Transportation after Preparation with Twisted File and Bio RaCe Instrumentation. J Dent (Tehran), 11, 440446.

Hulsmann M, P. O. (2005). Mechanical preparation of root canals: shaping goals, techniques and means. Endodontic Topics, 10, 30-76.

Lopes HP, F. A. (2009). Influence of rotational speed on the cyclic fatigue of rotary nickel-titanium endodontic instruments. J Endod, 35, 1013-1016.

Neelakantan P, R. P. (2016). Cyclic fatigue of two different single files with varying kinematics in a simulated doublecurved canal. J Investig Clin Dent, 7(3), 272-277.

Pedullà E, P. G. (2012). Cyclic fatigue resistance of four nickel-titanium rotary instruments. Annali di Stomatologia. 2012; III (2): 59-63. , 3(2), 59-63.

Pereira ESJ, P. I. (2012). Physical and mechanical properties of a thermomechanically treated NiTi wire used in the manufacture of rotary endodontic instruments. Int Endod J, 45, 469-474.

Perez-Higueras JJ, A. A. (2013). Cyclic fatigue resistance of K3, K3XF, and twisted file nickel-titanium files under continuous rotation or reciprocating motion. J Endod, 39, 1585-1588.

Pessoa OF, M. d. (2013). Cyclic fatigue resistance of rotary NiTi instruments after simulated clinical use in curved root canal. Braz Dent J, 24(2), 117-120.

Pongione G, P. G. (2012). Flexibility and resistance to cyclic fatigue of endodontic instruments made with different nickel-titanium alloys: a comparative test. Annali di Stomatologia, 3(3/4), 119-122.

Reddy PJ, K. V. (2014). Canal Shaping with One Shape File and Twisted Files: A Comparative Study. J Clin Diagn Res, 8, ZF01-ZF03.

Saleh AM, G. P. (2015). Shaping ability of 4 different single-file systems in simulated S-shaped canals. J Endod, 41(4), 548-552.

Schafer E, E. M. (2005). Influence of different types of automated devices on the shaping ability of rotary nickeltitanium FlexMaster instruments. Int Endod J, 38, 627-636.

Shen Y, Z. H. (2013). Current challenges and concepts of the thermomechanical treatment of nickel-titanium instruments. J Endod, 39, 163-172.

Specialties,D.T.(2015).ProtaperGOLD rotatory files Brochure. Retrieved from https://www.dentsply.com/content/dam/dentsply/pim/manufacturer/Endodontics/Glide_ Path_Shaping/Rotary_Reciprocating_Files/Shaping/ProTaper_Gold_Rotary_Files/ProTaper-Gold-Brochurep7btcwy-en-1502.pdf

Topçuoğlu HS, T. G. (2016). In vitro comparison of cyclic fatigue resistance of ProTaper Next, HyFlex CM, OneShape, and ProTaper Universal instruments in a canal with a double curvature. J Endod, 42(6), 969-971.

Wu H, P. C. (2015). Shaping ability of ProTaper Universal, WaveOne and ProTaper Next in simulated L-shaped and Sshaped root canals. . BMC Oral Health, 15, 27.

Wu M, F. B. (2000). Leakage Along Apical Root Fillings in Curved Root Canals. Part I: Effects of Apical Transportation on Seal of Root Fillings. J Endod, 26, 210-216.

Zupanc J, V. - P. N. (2018). New thermomechanically treated NiTi alloys - a review. Int Endod J, 51, 1088-1103. 